\title{
In Vitro Activity of Etanidazole against the Protozoan Parasite Trypanosoma cruzi
}

\section{Patricia B Petray $/{ }^{+} /++$, María J Morilla*/+++, Ricardo S Corral $/{ }^{++}$, Eder L Romero*}

Laboratorio de Virología, Hospital de Niños Dr. Ricardo Gutiérrez, Gallo 1330, C1425EFD, Buenos Aires, Argentina *Laboratorio de Diseño de Transportadores de Drogas, Universidad Nacional de Quilmes, Buenos Aires, Argentina

We investigated the in vitro action of an hydrosoluble 2-nitroimidazole, Etanidazole (EZL), against Trypanosoma cruzi, the etiologic agent of Chagas disease.

EZL displayed lethal activity against isolated trypomastigotes as well as amastigotes of T. cruzi (RA strain) growing in Vero cells or $J 774$ macrophages, without affecting host cell viability. Although not completely equivalent to Benznidazole (BZL), the reference drug for Chagas chemotherapy, EZL takes advantage in exerting its antiT. cruzi activity for longer periods without serious toxic side effects, as those recorded in BZL-treated patients. Our present results encourage further experiments to study in depth the trypanocidal properties of this drug already licensed for use in human cancers.

Key words: Trypanosoma cruzi - Etanidazole - chemotherapy

Chagas disease is widespreading throughout Latin America where nearly 20 million people are infected by Trypanosoma cruzi and 90 million are at risk in endemic areas (WHO 2000).

Current treatment for Chagas disease is dependent on the 2-nitroimidazole Benznidazole (Radanil, Roche), the only drug available for human use in Brazil and Argentina. This specific chemotherapy has limitations such as lack of effectiveness to achieve parasitologic cure or prevent the chronic phase of disease and emergence of parasite resistance (Urbina 1999). Moreover, the therapeutic dose is very close to the toxic dose, which turns this drug poorly tolerated, and severe side effects have been reported (Cançado 2002). In spite of this, the WHO (1999) has indicated that every $T$. cruzi-infected individual should be subjected to parasiticidal chemotherapy, independently from the stage of infection they undergo. It is worth to remark the lack of interest shown by pharmaceutical companies in protozoal infections given the high cost of drug development and limited - in value - market for these antiprotozoan drugs. In this context, there is a con-

Financial support: Swedish International Development Agency for the Network for Research and Training in Parasitic Diseases in the Southern Cone of Latin America and Vicerrectorado de Investigaciones de la UNQ

${ }^{+}$Corresponding author. Fax: +54-11-4962 6770. E-mail: ppetray@conicet.gov.ar

${ }^{++}$Member of the Research Career Programme from the Consejo Nacional de Investigaciones Científicas y Técnicas, Argentina ${ }^{+++}$Fellow from Comisión de Investigaciones Científicas de la Provincia de Buenos Aires, Argentina

Received 6 October 2003

Accepted 1 March 2004 siderable need for the development of new compounds to approach the chemotherapy for Chagas disease. In this work, we report the evaluation of in vitro trypanocidal activity of hydrosoluble Etanidazole (EZL, SR-2508), a new 2-nitroimidazole introduced in 1981 for use as a clinical hypoxic cell radiosensitizer (Coleman et al. 1984), which was proven to be less toxic in vivo compared to other evaluated 2-nitroimidazoles (Hurwitz et al. 1992).

EZL [N-(2-hydroxyethyl)-2-nitro-1-imidazoleacetamide] was obtained from NIH (US) and BZL (N-benzyl-2nitro-1-imidazoleacetamide) was provided by Roche Laboratories (Buenos Aires, Argentina) and was used as reference drug in every assay (Fig. 1). Stock solution of EZL was prepared in $10 \mathrm{mM}$ TRIS, $0.85 \% \mathrm{CINa}$, pH 7.4. BZL was solubilized in DMSO and the stock solution was prepared in $10 \mathrm{mM}$ TRIS, $0.85 \% \mathrm{CINa}, \mathrm{pH}$ 7.4. The final DMSO concentration in the experiments never exceeded $0.1 \%$ (vol/vol) and had no deleterious effect by itself on parasites or host cells.

T. cruzi trypomastigotes, RA strain, were grown and purified from monolayers of Vero cells cultured in RPMI1640 medium supplemented with $10 \%$ heat-inactivated fetal calf serum, $100 \mathrm{UI} / \mathrm{ml}$ penicillin and $100 \mu \mathrm{g} / \mathrm{ml}$ streptomycin (RPMI-10\%).

To evaluate the anti-trypomastigote activity, trypomastigotes $\left(10^{6} / \mathrm{ml}\right)$ were incubated in RPMI- $10 \%$ at $37^{\circ} \mathrm{C}$<smiles>O=C(Cn1ccnc1[N+](=O)[O-])NCCO</smiles><smiles>O=C(Cn1ccnc1[N+](=O)[O-])NCc1ccccc1</smiles>

Fig. 1: chemical structures of Etanidazole (a) and Benznidazole (b) 
in the presence or absence of EZL (14.8 to $1200 \mu \mathrm{M}$ ) or BZL (1.48 to $120 \mu \mathrm{M})$. After 24 or 48 h-culture, reduction in $T$. cruzi number (parasite lysis) was determined using a Neubauer chamber. The $\mathrm{LD}_{50}$ value (drug concentration that resulted in a 50\% reduction compared to the untreated control) was estimated by plotting percentage of reduction against the logarithm of drug concentration.

Assays of anti-amastigote activity were performed in Vero cells or J774 murine macrophages. Cells were seeded at a density of 50,000 cells/well in 24-well microplates with rounded coverslips on the bottom. After infection at a parasite-to-cell ratio of $10: 1$ for $24 \mathrm{~h}$ at $37^{\circ} \mathrm{C}$, extracellular parasites were removed by six washes with PBS, $\mathrm{pH} 7.2$, and fresh medium containing EZL ( 3 to $243 \mu \mathrm{M}$ ), BZL ( 0.3 to $24.3 \mu \mathrm{M}$ ) or none was added. Seventy two hours later, the coverslips were fixed and stained with Giemsa and the number of amastigotes/100 cells was calculated by use of light microscopy. Anti-amastigote activity was expressed as: $\% \mathrm{AA}=[1-(\mathrm{nr}$ amast $/ 100$ cells $)$ treated $/(\mathrm{nr}$ amast $/ 100$ cells)control] x 100 . The $\mathrm{IC}_{50}$ was defined as the drug concentration required to achieve $50 \%$ inhibition of intracellular amastigote growth.

Cytotoxicity assays were carried out on Vero cells or J774 murine macrophages seeded in 96-well flat bottom microplates $\left(10^{5}\right.$ cells/well) in $200 \mu$ of RPMI- $10 \%$. The cells were allowed to attach for $24 \mathrm{~h}$ at $37^{\circ} \mathrm{C}$ and then exposed to EZL ( 3 to $243 \mu \mathrm{M}$ ) or BZL ( 0.3 to $24.3 \mu \mathrm{M}$ ) for

a

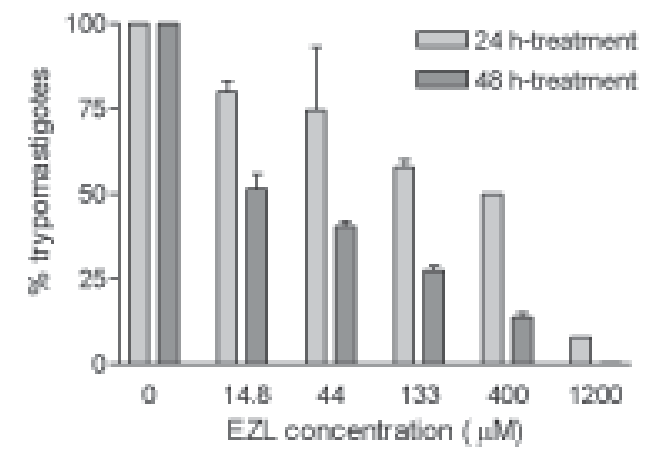

c

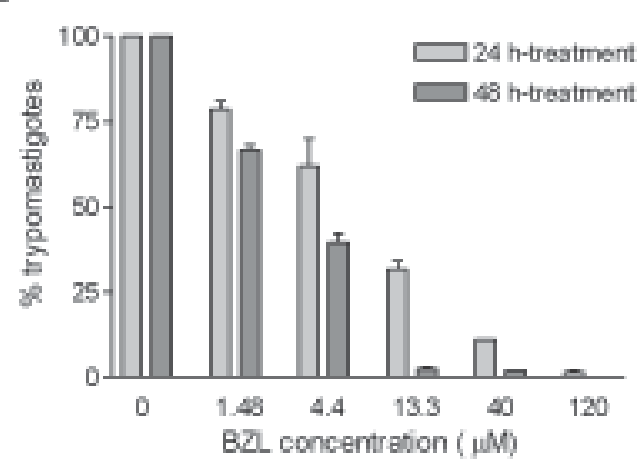

$72 \mathrm{~h}$. After incubation, the cells were washed with PBS and incubated with sodium 3'-[1-(phenylamino-carbonyl)3,4-tetrazolium]-bis-(4-methoxy-6-nitro) benzene sulfonic acid hydrate (XTT) $200 \mu \mathrm{g} / \mathrm{ml}$ for $4 \mathrm{~h}$. XTT solution was removed and the extent of reduction of XTT to formazan within the cells was quantified by measurement of spectrophotometrical absorbance at $450 \mathrm{~nm}$ using an ELISA reader (Meletiades et al. 2001). Each concentration was assayed three times and six cell growth controls were used for each test.

As shown in Fig. 2a, EZL treatment provoked a dosedependent reduction in the number of RA trypomastigotes of T. cruzi, with the most remarkable effect achieved at 48 h. EZL was again notably active against the clinically relevant intracellular amastigote forms of $T$. cruzi. Indeed, amastigotes infecting Vero cells or J774 macrophages were more susceptible to the new compound than trypomastigotes (Fig. 2b). At the observation timepoint after infection (72 h), only intracellular forms of the parasite could be seen in drug-treated cell cultures. No change in the viability of Vero cells or J774 macrophages could be observed even at the highest concentration of the drugs tested, indicating that T. cruzi and not host cells is the target for EZL (Fig. 2b, d).

Our results (Fig. 2c, d) showed that EZL [ $\mathrm{LD}_{50}$ values from $174 \mu \mathrm{M}(24 \mathrm{~h})$ to $18 \mu \mathrm{M}(48 \mathrm{~h})$, for trypomastigotes and $\mathrm{IC}_{50}$ values from $40 \mu \mathrm{M}$ (in Vero cells) to $15 \mu \mathrm{M}$ (in

b
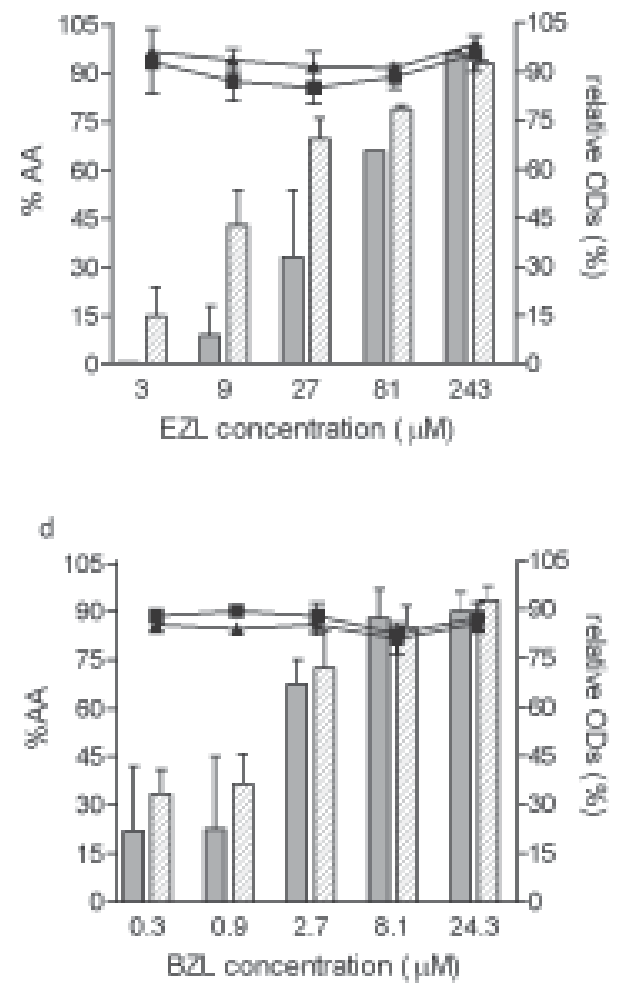

Fig. 2: activities of Etanidazole (EZL) and Benznidazole (BZL) against Trypanosoma cruzi. a and c: lysis of trypomastigote forms of $T$. cruzi determined in the presence of EZL (a) or BZL (c) at different drug concentrations; b and d: activities of EZL (b) and BZL (d) (72 htreatment) against intracellular T. cruzi amastigotes infecting either Vero cells (solid bars) or J774 murine macrophages (hatched bars) and drug effect on growth of Vero $(\boldsymbol{\square})$ or J774 ( $\boldsymbol{\Delta})$ cell types. Relative ODs for each concentration represent the amount of produced formazan compared with the growth control; \% AA: anti-amastigote activity. Results represent mean values \pm SEM for one representative of two similar experiments. 
J774 macrophages) for amastigotes] is less potent than BZL $\left[\mathrm{LD}_{50}\right.$ values from $6.6 \mu \mathrm{M}(24 \mathrm{~h})$ to $2.2 \mu \mathrm{M}(48 \mathrm{~h})$ for trypomastigotes and $\mathrm{IC}_{50}$ values from $1.7 \mu \mathrm{M}$ (Vero) to $1 \mu \mathrm{M}(\mathrm{J} 774)$ for amastigotes] in eliminating parasites. It is likely that prolonged in vivo administration of EZL should be required to achieve a trypanocidal activity equivalent to that exhibited by BZL. Nevertheless, this may not be a disadvantage for the clinical application of this compound taking into account that, compared to other evaluated 2nitroimidazoles, EZL has demonstrated to be less neurotoxic in vivo (Coleman et al. 1984). Undesired toxic side effects have always been considered a serious drawback for BZL chemotherapy in Chagas patients (Cançado 2002).

In this study, EZL was effective in vitro against the highly virulent RA strain of T. cruzi. However, its parasiticidal action should also be examined against other strains of the parasite, at the light of reported failures of chemotherapy in eliminating some T. cruzi isolates (Filardi \& Brener 1987).

Although the mechanism of trypanocidal action of EZL remains to be elucidated, previous studies have indicated that under normal oxygen tension EZL experiences a futile redox cycling that generates reactive oxygen species (ROS) toxic for the parasites. However, under hypoxic conditions, EZL is reduced by oxygen-sensitive nitroreductases to free radical species which form adducts with a series of macromolecules. On the other hand, under normal oxygen tension BZL seems to be reduced by putative oxygen-insensitive nitroreductases, and many works have found its reduction products covalently bound both to host and parasite moieties, whereas there is no generation of ROS nor futile redox cycling experienced (Lord et al. 1993). Hence, it is expected that both 2-nitroimidazoles metabolize and exert their parasiticidal action by different pathways.

In conclusion, we have found out a completely novel and different application for EZL, demonstrating that this 2-nitroimidazole displays a defined anti-T. cruzi activity against both circulating and intracellular stages of the parasite. Though EZL presented higher $\mathrm{LD}_{50}$ and $\mathrm{IC}_{50}$ than those corresponding to BZL, the reference drug for treatment of chagasic patients, its known lack of significant toxicity in human antitumor therapy (Coleman et al. 1984, Wasserman et al. 1991, Riese et al. 1994, Marcus et al. 2003) supports further investigations, including in vivo evaluation, to establish the real potential of this compound as chemotherapeutic agent against Chagas disease.

\section{REFERENCES}

Cançado JR 2002. Long term evaluation of etiological treatment of Chagas' disease with benznidazole. Rev Inst Med Trop São Paulo 44: 29-37.

Coleman CN, Urtasun RC, Wasserman TH, Hancock S, Harris JW, Halsey J, Hirst VK 1984. Initial report of the phase I trial of the hypoxic cell radiosensitizer SR-2508. Int J Radiat Oncol Biol Phys 10: 1749-1753.

Filardi LS, Brener Z 1987. Susceptibility and natural resistance of Trypanosoma cruzi strains to drugs used in Chagas' disease. Trans R Soc Trop Med Hyg 81: 755-759.

Hurtwitz SJ, Coleman CN, Riese N, Loeffler JS, Alexander E, Buswell L, Neben TY, Shargel L, Kramer RA 1992. Distribution of Etanidazole into human brain tumors-implications for treating high grade gliomas. Int J Radiat Oncol Biol Phys 22: 573-576.

Lord EM, Harwell L, Koch CJ 1993. Detection of hypoxic cells by monoclonal antibody recognizing 2-nitroimidazole adducts. Cancer Res 53: 5721-5726.

Marcus KJ, Dutton, MPH, Barnes P, Coleman CN, Pomeroy SL, Goumnerova L, Billett AL, Kieran M, Tarbell NT 2003. A phase I trial of etanidazole and hyperfractionated radiotherapy in children with diffuse brainstem glioma. Int $J$ Radiat Oncol Biol Phys 55: 1182-1185.

Meletiades J, Mouton, JW, Meis JFGM, Bouman BA, Donnelly JP, Verweij PE, Eurofund Network 2001. Colorimetric assay for antifungal susceptibility testing of Aspergillus species. J Clin Microbiol 39: 3402-3408.

Riese NE, Loeffler JS, Wen P, Alexander E, Black PM, Coleman CN 1994. A phase I study of etanidazole and radiotherapy in malignant glioma. Int J Radiat Oncol Biol Phys 29: 617620.

Urbina JA 1999. Chemotherapy of Chagas' disease: the how and the why. J Mol Med 77: 332-338.

Wasserman TH, Lee DJ, Cosmatos D, Coleman N, Phillips T, Davis L, Marcial V, Stetz J 1991. Clinical trials with etanidazole (SR-2508) by the Radiation Therapy Oncology Group (RTOG). Radiother Oncol 20 (Suppl.1): 129-135.

WHO 1999. Technical Report Series. Document OPS/ HCP/ HCT/140.

WHO 2000. Special Programme for Research and Training in Tropical Disease (TDR). Natural products for parasitic diseases. TDR News 62: 4. 